In der Rubrik "Literatur kompakt" dieser Ausgabe referieren und kommentieren diese Experten für Sie Arbeiten aus der internationalen Fachliteratur.

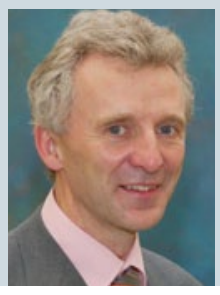

Dr. med. Martin Claßen, Bremen

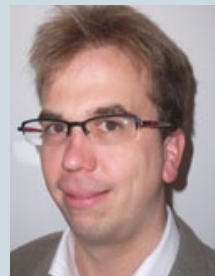

Prof. Dr. med. Helge Frieling, Hannover

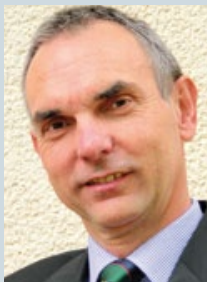

Prof. Dr. med. Adrian Gillissen, Kassel

\section{Gewalt gegen behinderte Kinder kommt häufig vor}

Naiverweise glauben viele Menschen, dass behinderte Kinder wegen ihrer offensichtlichen Schutzbedürftigkeit seltener Opfer von Gewalt werden als ihre nicht behinderten Altersgenossen. Diese Annahme ist leider falsch.

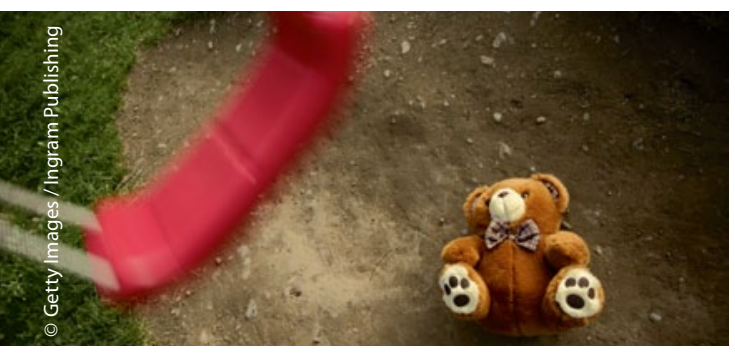

— ine Arbeitsgruppe aus Liverpool hat — eine systematische Untersuchung und Metaanalyse unter Benutzung von zwölf elektronischen Datenbanken für die Zeit von 1990 bis 2010 angestellt. Ihr Ziel war es, die Prävalenz von Gewalt gegenüber Kindern unter 18 Jahren mit Behinderungen festzustellen bzw. das Risiko, Opfer einer Gewalttat zu werden, verglichen mit Kindern ohne Behinderung. Es wurden insgesamt 16 Studien für die Metaanalyse von Prävalenzdaten ausgewertet und elf für die Risikodaten.

Die kombinierte Prävalenzhäufigkeit von physischer und emotionaler Gewalt betrug $26,7 \%$ - für physische Gewalt alleine $20,4 \%$ und für sexuelle Gewalt 13,7\%. Die Wahrscheinlichkeit eines behinderten Kindes, Opfer physischer Gewalt zu werden, war damit etwa dreieinhalbmal so hoch wie für ein nicht-behindertes Kind. Die Wahrscheinlichkeit Opfer sexueller Gewalt zu werden, war für ein Kind mit Behinderungen fast dreimal so hoch wie für ein nicht-behindertes.
Rund ein Viertel aller behinderten

Kinder macht im Laufe ihres Lebens die Erfahrung einer Gewalttat.

Die Autoren schließen aus diesen Ergebnissen, dass bis zu einem Viertel aller behinderten Kinder im Laufe ihres Lebens die Erfahrung einer Gewalttat machen wird. Sie folgern weiter, dass behinderte Kinder als Hochrisikogruppe für
D er Einfluss einer chronischen Feinstaubexposition auf die Lungengesundheit von Kindern ist in mehreren Studien untersucht worden - wobei die Ergebnisse sehr unterschiedlich ausfielen. Für die vorliegende Auswertung wurden Daten mehrerer epidemiologischer Studien aus zwölf Ländern in Nordamerika und Europa herangezogen. Analysiert wurden Fragebogendaten zur Beschwerdesymptomatik von 45.788 Kindern. Bei etwa der Hälfte der Patienten lagen auch Lungenfunktionswerte vor.
Opfer von Gewalttaten gelten müssen.

Jones $L$ et al. Prevalence and risk of violence against children with disabilities: a systematic review and meta-analysis of observational studies. Lancet 2012; 380: 899-907

Kommentar: Das Ergebnis dieser Studie ist leider vollkommen anders, als man selbst es naiverweise erwartet hätte. Möglicherweise wären die Zahlen noch höher, wenn es Studien aus Ländern mit geringem oder mittlerem Einkommen gäbe. Unklar bleibt auch, bei wie vielen Kindern die Gewaltanwendung Ursache der Behinderung war. Für den Kinderarzt bleibt wichtig, dass die Schutzbedürftigkeit behinderter Kinder offensichtlich höher ist als angenommen. Dr. Hartmut Koch

\title{
Chronische Feinstaubbelastung führt zu Atembeschwerden bei Kindern
}

\section{Der Nachweis eines Zusammenhangs zwischen Umweltschadstoffen und Lungenerkrankungen ist mühsam. Nun zeigt eine Metaanalyse einen signifikanten Zusammenhang zwischen chronischer Feinstaubbelastung und Atemwegsbeschwerden bei Kindern.}

Die höchsten Feinstaubkonzentrationen (Partikelgröße unter $10 \mu \mathrm{m}, \mathrm{PM} 10)$ wurden in den osteuropäischen Ländern nachgewiesen. Nach Adjustierung für diverse Störfaktoren ergab sich ein signifikanter Zusammenhang zwischen chronischer Feinstaubbelastung und den Symptomen Auswurf sowie morgendlicher Husten. Der Zusammenhang zur Arztdiagnose Bronchitis, zu nächtlichem Husten und zu Heuschnupfen war ebenfalls noch schwach signifikant positiv. Es ergab sich aber 